\title{
X-Ray Diffraction Studies on Liquids at Very High Pressures along the Melting Curve II. Sodium
}

Keith H. Brown

J. Dean Barnett

jdeanbarnett@gmail.com

Follow this and additional works at: https://scholarsarchive.byu.edu/facpub

Part of the Astrophysics and Astronomy Commons, and the Physics Commons

\section{Original Publication Citation}

Brown, Keith H. and Dean J. Barnett. "X-Ray Diffraction Studies on Liquids at Very High Pressures along the Melting Curve II. Sodium." The Journal of Chemical Physics 57 (1972): 216-221.

\section{BYU ScholarsArchive Citation}

Brown, Keith H. and Barnett, J. Dean, "X-Ray Diffraction Studies on Liquids at Very High Pressures along the Melting Curve II. Sodium" (1972). Faculty Publications. 788.

https://scholarsarchive.byu.edu/facpub/788 


\title{
X-Ray Diffraction Studies on Liquids at Very High Pressures along the Melting Curve II. Sodium*
}

\author{
Keith H. Brown $†$ and J. Dean Barnett \\ Department of Physics, Brigham Young University, Provo, Utah 84601
}

(Received 2 March 1972)

\begin{abstract}
Coherent $\mathrm{x}$-ray diffraction patterns for liquid $\mathrm{Na}$ have been extracted from measured patterns at 0.5 , $7.5,17$, and $43 \mathrm{kbar}$ along the melting curve. The experimentally determined liquid structure factor and resulting radial distribution function are given at each pressure. The position of the principal $x$-ray intensity peak is reproducible to within $0.5 \%$ and shows shifts of the order of $12 \%$. The resulting nearest neighbor distance in the radial distribution function also shifts $12 \%$ but is reproducible only to approximately $2 \%$. The distribution functions obtained at points along the melting curve suggest a simple dimensional compression or scaling; however, a sharpening of the distribution peaks slightly in excess of that predicted by simple scaling is indicated by the measurements.
\end{abstract}

\section{INTRODUCTION}

A technique for using $\mathrm{x}$-ray diffraction to investigate the structure of liquids along the melting curve to high temperatures and pressures has been discussed in Paper I of this series. ${ }^{1}$ This technique has been used to determine the coherent $x$-ray intensity pattern and the radial distribution function of sodium at the four points along the melting curve ${ }^{2}$ shown in Fig. 1. The uncertainty in pressure is indicated.

Sodium was chosen as the first material to be investigated for the following three reasons: (a) It is a simply structured monatomic liquid which has been widely studied experimentally and theoretically at ordinary temperatures and 1 bar pressure in both the liquid and the solid states. (b) Since it is highly compressible, large changes are expected in the radial distribution function as high pressures are exerted on the sample. (c) It is experimentally convenient since it is solid at room temperature, has a low melting temperature, and has a relative low $\mathrm{x}$-ray absorption coefficient. Both theoretical ${ }^{3}$ and experimental ${ }^{4}$ equations of state are available, which fact allows one to estimate the density of the liquid near the melting curve. Furthermore, when experimental information is available on the liquid state, correlations can be made with the solid to elucidate the fundamental theory of melting. In this paper, however, no attempt will be made to make such correlations.

$\mathrm{X}$-ray and neutron diffraction have been used to investigate liquid sodium by a number of different investigators. ${ }^{5-9}$ The results of these studies suggest that with increasing temperature the coordination number decreases slightly and the first peak in the radial distribution function shifts slightly to lower $r$ values. Similar but larger changes in the RDF are expected with high pressure.

\section{EXPERIMENTAL TECHNIQUES FOR SODIUM}

The basis sample design is described in Sec. II of Paper I of this series. The tetrahedrons of $50-50 \mathrm{wt} \%$ boron-plastic mixture were made for use with $3 / 4$ in. anvils. The sodium was placed in an aluminum cell to protect it from chemical contamination and to contain the molten sodium. The sodium was initially
99.9\% pure and was placed in the aluminum cell in a dry box with an argon atmosphere. It is difficult to ascertain how much contamination of the sodium occurred during preparation and while measurements were being taken, but the x-ray pattern did not show the existence of large amounts of impurities.

The temperature was held constant to within $1^{\circ} \mathrm{C}$ by the use of a temperature controller designed by Decker. ${ }^{10} \mathrm{X}$-ray diffraction provides a means of directly determining when melting occurs. The melting temperature measurement was generally repeatable to within $3^{\circ} \mathrm{C}$ for a given sample. The difference between the temperature where a crystalline $x$-ray pattern was observed and where a smooth, broad, liquid peak was observed was generally less than $2^{\circ} \mathrm{C}$, which result implies that the temperature gradients within the sample were less than $2^{\circ} \mathrm{C}$. Temperature gradients in the tetrahedron but outside the sodium metal introduce uncertainties of as much as $10-15^{\circ} \mathrm{C}$ in the absolute temperature determination by the chromel-alumel thermocouple. If the pressures are independently known this uncertainty is of no consequence.

Pressure calibration based on $\mathrm{NaCl}$ compressibility ${ }^{11}$ was made using a calibration of the load applied to the hydraulic rams when using a similar type sample at a specific temperature. The estimated accuracy of the pressure values is $8 \%$. The pressure values used are consistent with values estimated using the measured temperatures and referring to the known melting curve. ${ }^{2}$

\section{X-RAY INTENSITY PATTERNS}

Normalized coherent $\mathrm{x}$-ray intensity patterns were obtained using the technique developed in Paper I of this series. Briefly, this technique involves four preliminary steps: (a) X-ray patterns are obtained at a given pressure of the sodium while it is a liquid and while it is a solid; (b) the two patterns are subtracted to eliminate background scattering; (c) the resulting difference pattern is corrected for absorption, polarization, and incoherent scattering; (d) 8-10 such patterns are combined and smoothed to give average curves which are called composite patterns. 
FIG. 1. The four points along the melting curve of $\mathrm{Na}$ at which coherent $\mathrm{x}$-ray intensity patterns and radial distribution functions were determined. The uncertainty in pressure at each point is indicated.

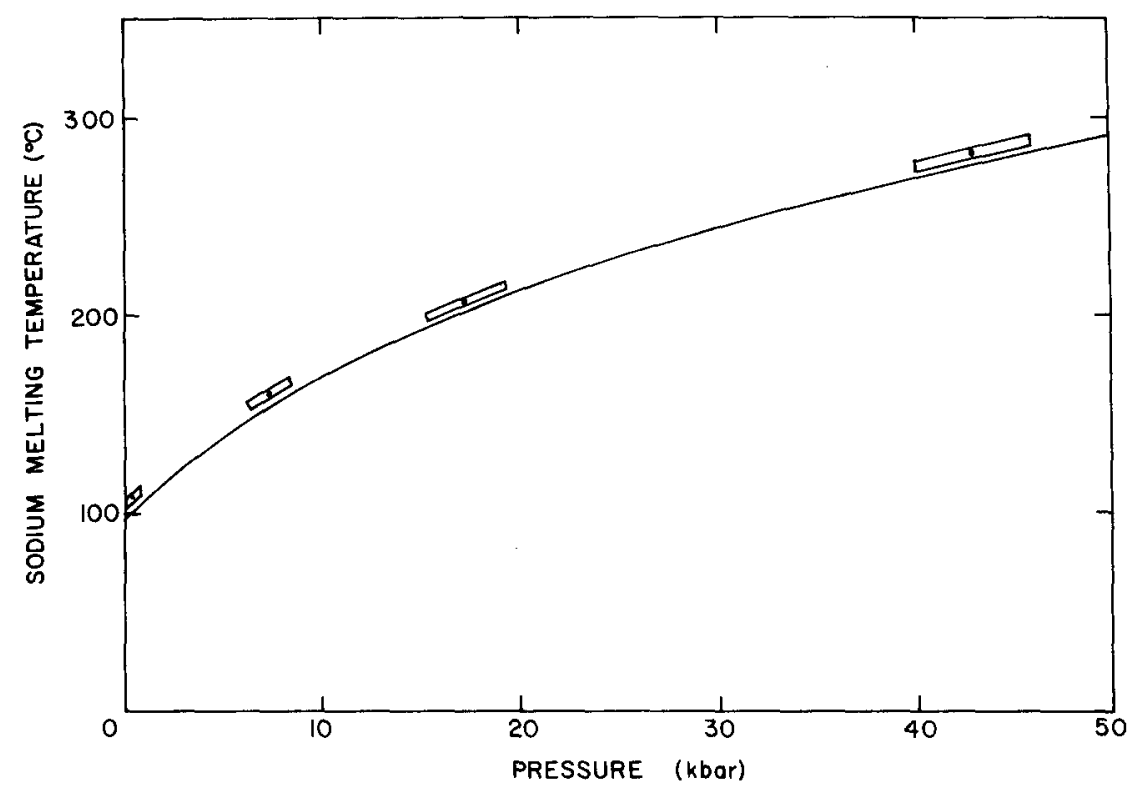

The composite pattern $I^{\text {omp }}$ represent the difference, $I_{1 \mathrm{iq}}{ }^{\mathrm{oh}}-I_{\mathrm{Bol}}{ }^{\mathrm{TDS}}$, between the coherent diffracted intensity from the liquid and the thermal diffuse scattering from the solid in the vicinity of melting. The composite patterns obtained at $0.5,7.5,17$, and $43 \mathrm{kbar}$ are shown in Fig. 2.

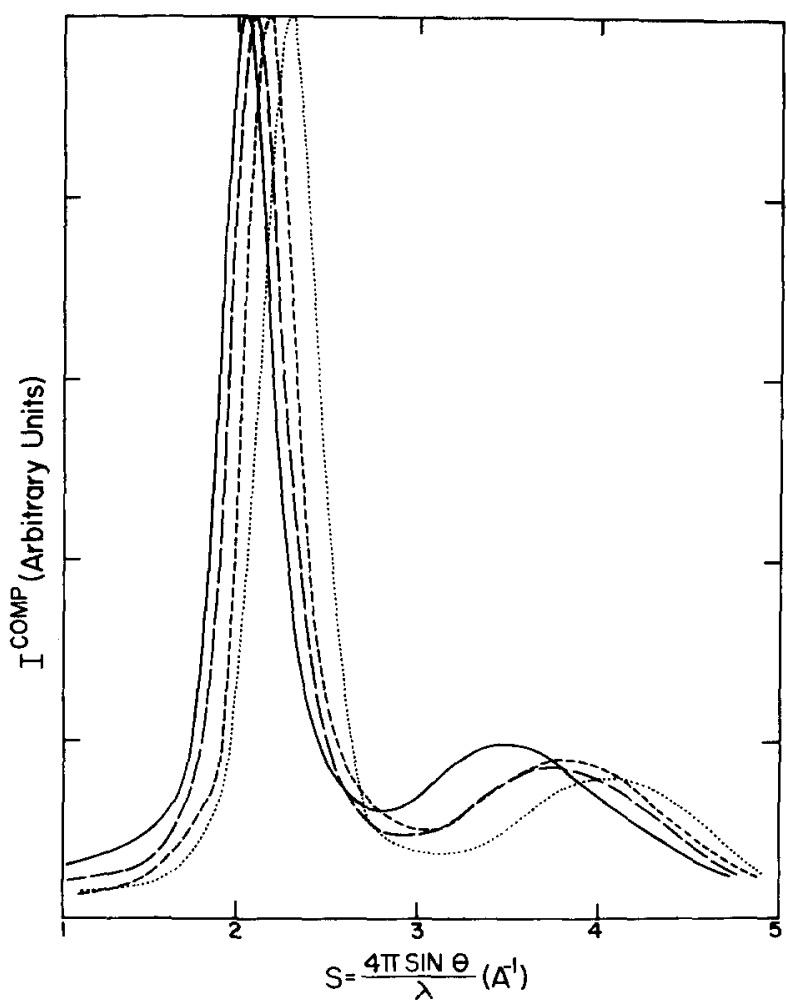

Fig. 2. Composite patterns, $I^{\circ 0 \mathrm{mp}}=I_{1 \mathrm{coh}}-I^{\mathrm{osl}} \mathrm{TDB}$, for $\mathrm{Na}$ at pressures of (a) $0.5 \mathrm{kbar},-$; (b) $7.5 \mathrm{kbar},--$; (c) $17 \mathrm{kbar}$, $\cdots$, and (d) 43 kbar, $\cdots$.
The normalized coherent $x$-ray intensity pattern for the liquid is obtained by normalizing $I^{\text {comp }}$ as discussed in Sec. $V$ of Paper I and by then adding a theoretical estimate of the thermal diffuse scattering component. A normalized coherent pattern for each pressure is shown in Fig. 3. The position of the first peak in the intensity function at each pressure is well defined and shows consistent change to larger $S(S=4 \pi \sin \theta / \lambda)$ with increasing pressure. The second peak is not so well defined but still shows some consistent change to larger $S$ with pressure.

The consistency of measurement of the first peak in the intensity pattern at various pressures along the melting curve is indicated by Fig. 4 . Numerous patterns were obtained at pressures of $0.5,7.5,17$, and $43 \mathrm{kbar}$; so the average values shown for peak positions at these pressures should be reliable. A few $\mathrm{x}$-ray patterns were also obtained at pressures of 3.5 , $9,27.5$, and $54 \mathrm{kbar}$. These patterns provide supporting data about the variation of the intensity pattern with pressure. The composite patterns obtained at the former four pressures were the ones used to obtain the radial distribution functions, but not enough patterns were obtained at the latter pressures to make a meaningful determination of the RDF possible. The diffraction angle error flags designate two times the error of the mean, i.e., $2 \sigma / n^{1 / 2}$, where $\sigma$ is the standard deviation from the mean of $n$ repeated measurements at a given pressure. Location of the first peak is shown at $13.28^{\circ}$ at $1 \mathrm{bar}$, which value is the average of four values from the literature. ${ }^{5-8}$

\section{RADIAL DISTRIBUTION FUNCTIONS}

By following a standard technique outlined in $\mathrm{Pa}$ per $I$ of this series, the radial distribution function, $\mathrm{RDF}=4 \pi r^{2} \rho(r)$, has been obtained from the normalized 


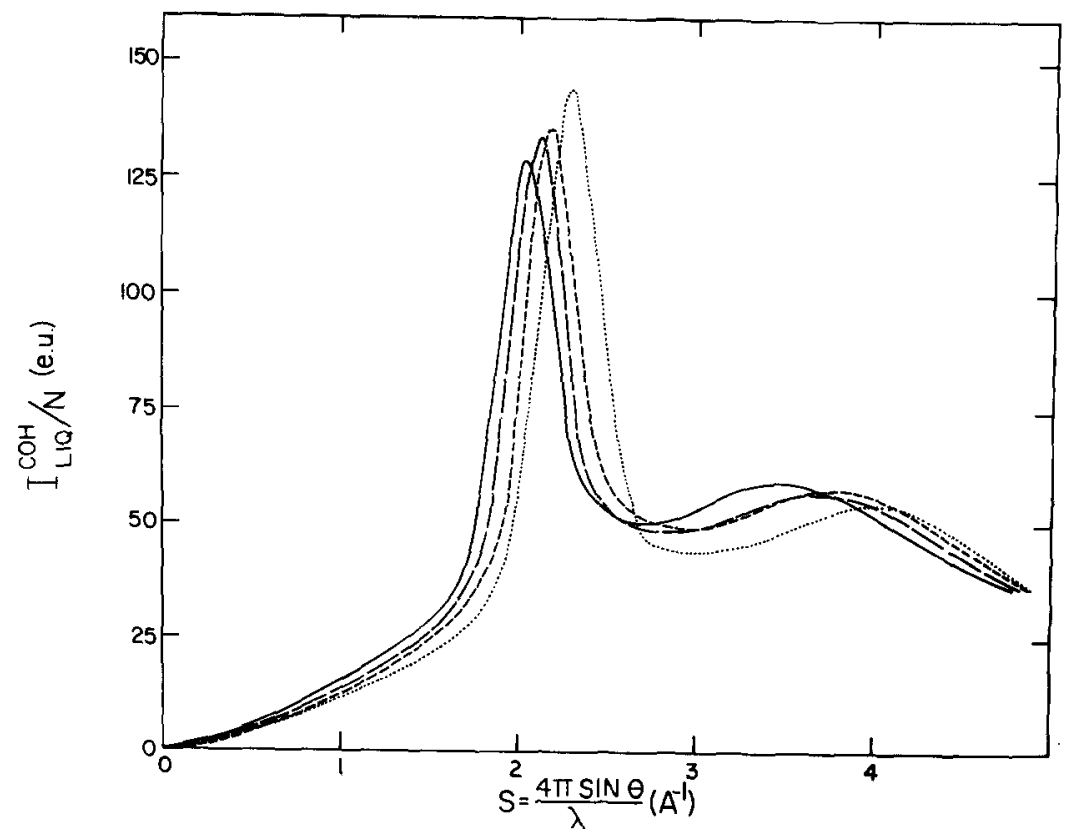

FIG. 3. Normalized coherent $\mathrm{x}$-ray intensity, $I_{\mathrm{liq}}{ }^{\mathrm{cob}} / N$, for $\mathrm{Na}$ at pressures of (a) $0.5 \mathrm{kbar},-$; (b) $7.5 \mathrm{kbar},--$; (c) 17 kbar, - . - ; and (d) 43 kbar, .... coherent $x$-ray intensity and involves a knowledge of the average density $\rho_{0}$ and the atomic scattering factor $f^{2}$ at pressure. The average density of the solid at each point along the melting curve was estimated from the theoretical equation of state for solid sodium proposed by Pastine. ${ }^{3}$ Since the density change upon melting is only about $2.5 \%$ at atmospheric pressure and would be expected to decrease at higher pressures, the density of the liquid can be estimated quite accurately using the technique outlined in Sec. VI of Paper I of this series. The atomic scattering factors calculated by Cromer and Waber ${ }^{12}$ were used. Further work by Cromer $^{13}$ has shown that $f^{2}$ does not vary significantly over the range of pressures in this experiment.

The RDF obtained at $0.5,7.5,17$, and $43 \mathrm{kbar}$ along the melting curve are shown in Fig. 5. The position of the first peak and the area under the peak represent interesting quantitative features of the liquid structure. The ripple at small values of $r$ (less than about $2.5 \AA$ ) arises from errors in the determination of these RDF. The ripple may be caused by errors in the absorption correction, atomic scattering factor values, determination and normalization of the coherent $x$-ray intensity, or errors introduced by terminating at too low a diffraction angle. The amplitude of the ripple is approximately twice that obtained by other investigators at atmospheric pressure. The observed amplitude of ripple is not unreasonable considering the sensitivity of the ripple to the factors listed above and the severe limitations imposed by the pressure system.

One immediately notices from Fig. 5 the consistent variation in the position of the first principal peak in the RDF with pressıre. Numerical values for these positions are $3.89,3.67,3.58$, and $3.44 \AA$ at pressures of $0.5,7.5,17$, and $43 \mathrm{kbar}$ respectively. These peak positions correspond to the average nearest neighbor distance in the liquid. As expected, the nearest neighbor distance decreases as one goes to higher pressures

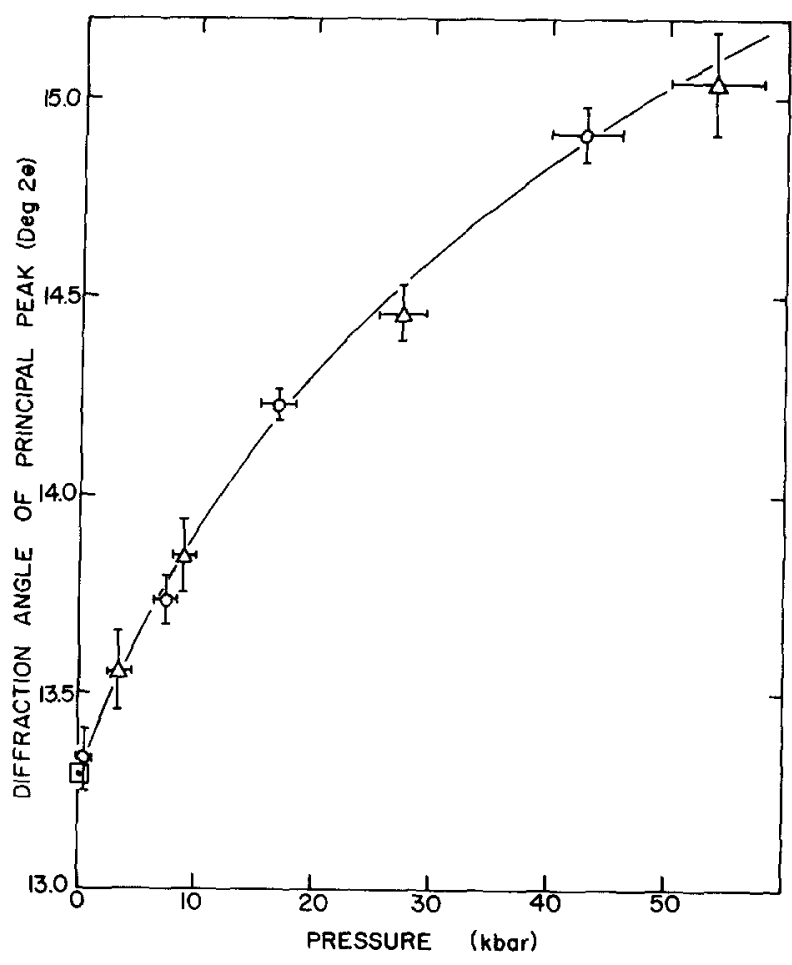

FIG. 4. Measured values of the angular position of the first peak in the $x$-ray intensity, $I^{\mathrm{com} D}$, as a function of pressure: $O$ points from patterns used to calculate the RDF, $\triangle$ supplementary points, $\square$ average value from the literature at 1 bar. 
along the melting curve. The width of the first peak tends to decrease, and the height increases with pressure. Calculations by the symmetric peak method of the area under the peak, which represents the coordination number, show a variation of less than $6 \%$ along the melting curve. This variation is less than the uncertainty expected due to errors in the analysis and is not a consistent variation with pressure.

The second peak in the radial distribution function contains information about the second nearest neighbors. However, this peak is so highly susceptible to errors in the analysis process that in much of the work done at atmospheric pressure some reluctance is found in placing great reliability on it. Because of the increased errors involved in analysis at high pressure, little confidence can be placed in details about the second principal peak. Qualitatively Fig. 5 indicates that the second peak also moves to smaller values of $r$ with increasing pressure, but quantitative values are surely not reliable.

\section{v. DISCUSSION AND CONCLUSION}

In analyzing the effects of pressure on liquid structure, we are primarily interested in two conceptually independent changes: (a) simple compression in which the atoms maintain the same time-average positions

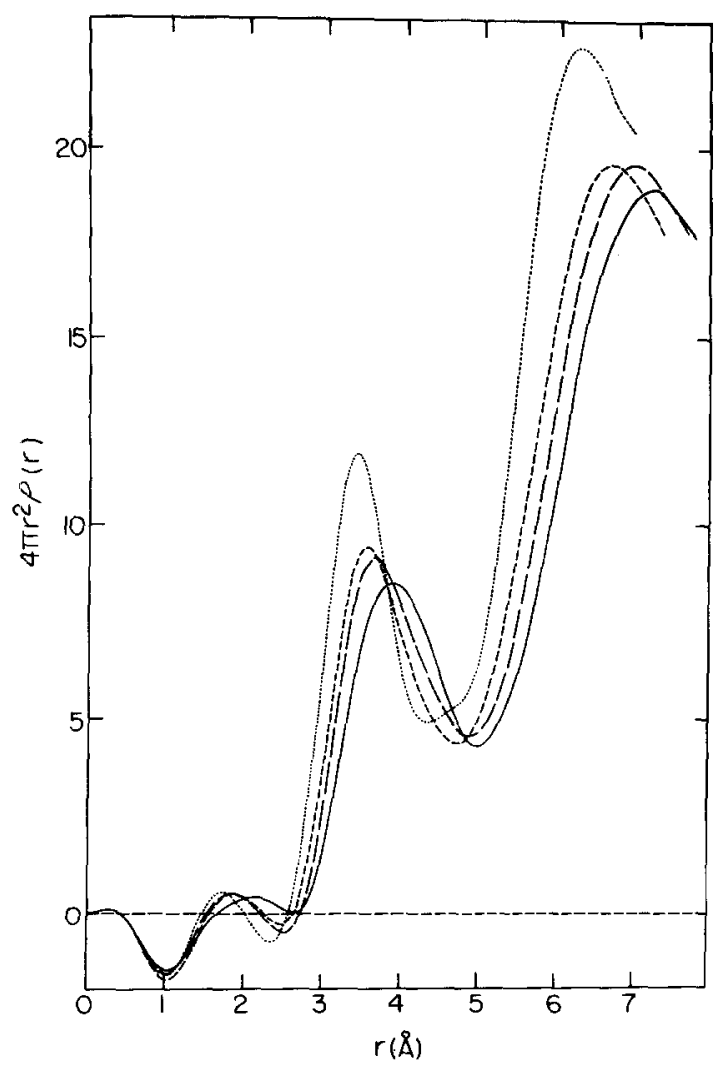

Fig. 5. Radial distribution functions, $\mathrm{RDF}=4 \pi r^{2} \rho(r)$, for $\mathrm{Na}$ at pressures of (a) $0.5 \mathrm{kbar},-$; (b) $7.5 \mathrm{kbar},--$; (c) $17 \mathrm{kbar}$, $\cdots$; and (d) 43 kbar, $\cdots$.

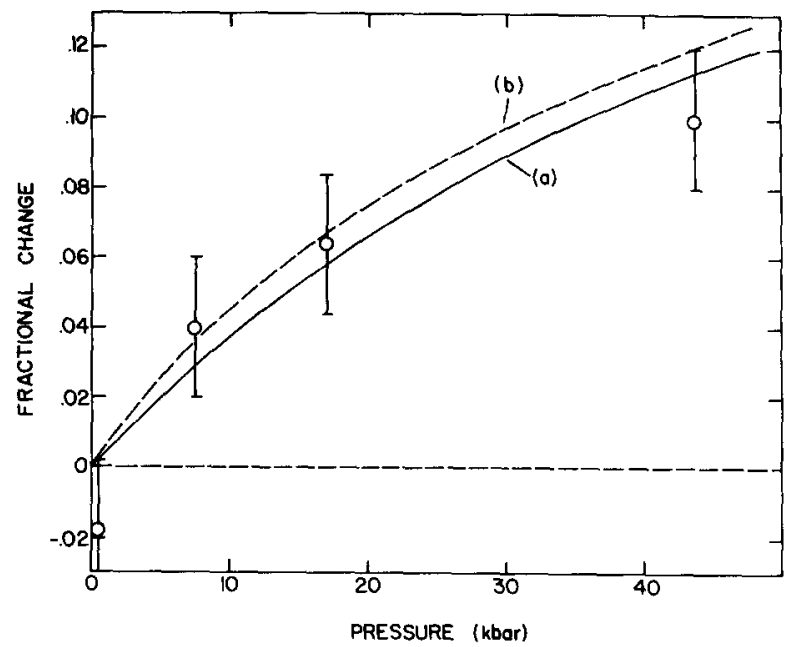

Fig. 6. Comparison of experimental values of the fractional change in the nearest neighbor distance, $\Delta R / R(0)$, at four pressures along the melting curve with curve (a), the fractional change in an average interatomic distance, $\Delta \bar{R} / \bar{R}$, associated with the estimated volume, and with curve (b), the fractional change in the scaling parameter, $\Delta a / a$, calculated from the measured dominant intensity peak positions.

relative to each other, i.e., the same ordering, and (b) ordering changes in which the relative distances may change (which corresponds to a change in the packing fraction for the hard-sphere model). The change (a) represents a simple dimensional contraction in real space commonly called scaling. Changes in ordering can best be detected by determining departures from simple scaling. The simple scaling concept is of special interest since it has been used ${ }^{14}$ in theoretical treatments of liquids and melting.

\section{Nearest Neighbor Distance}

The most dominant feature observed in the RDF patterns in Sec. IV is the general, consistent shift of the patterns to smaller $r$ with pressure, which shift suggests that scaling is a significant effect in liquid sodium along the melting curve. Values of the fractional changes in nearest neighbor distance, $\Delta R / R(0)$, at the four specified pressures along the melting curve are shown in Fig. 6. The $R(0)$ value used in calculating these points was the average of the literature values $^{5-9}$ at atmospheric pressure $(3.82 \AA)$. The error flags were estimated by making various changes in the intensity corrections and analysis procedures.

Two curves are also shown in Fig. 6 for comparison. Curve (a) gives changes $\Delta \bar{R} / \bar{R}$ based on a volumetric argument as follows: If the volume is known, changes in the average interatomic distance $\bar{R}$ can be calculated by the dimensional argument that $\bar{R}^{3}$ is proportional to $V$. Good estimates of the relative volume of the liquid near the melting curve can be made using the theoretical equation of state of solid sodium by Pastine, ${ }^{3}$ the Clausius-Clapeyron equation, and by assuming the change in entropy due to melting is 


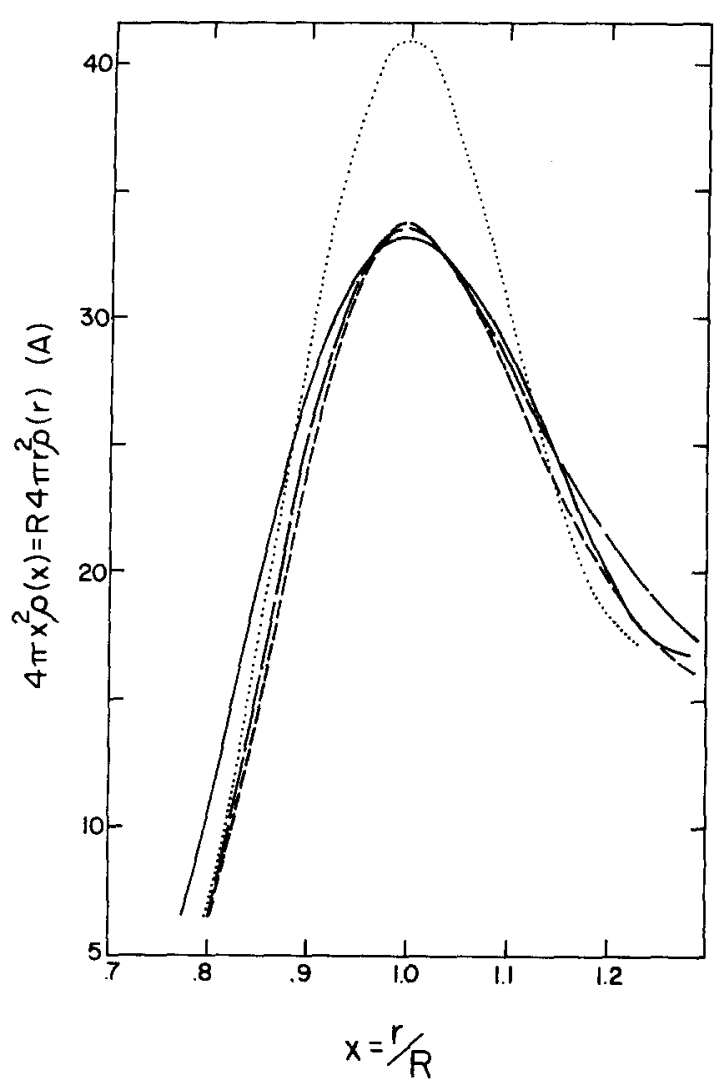

Fig. 7. The first peaks of the scaled RDF where the position of the first peak in the unscaled RDF is used as a scaling factor, illustrating departure from simple scaling. The RDF are for Na at pressures of (a) $0.5 \mathrm{kbar},-$; (b) $7.5 \mathrm{kbar},--$; (c) $17 \mathrm{kbar}$, $\cdots$ - ; and (d) $43 \mathrm{kbar}, \cdots$.

approximately constant along the melting curve. Since the volume change across melting is of the order of $2 \%$, an error of $50 \%$ in the entropy change would produce a volume error which is small in comparison to the changes in volume produced by pressure. Curve (b) of Fig. 6 represents a fraction change $\Delta a / a$ in linear dimension determined from the position of the dominant peak in the intensity pattern under the assumption that the total effect of pressure on the liquid along the melting curve is a simple dimensional scaling in real space. Assuming such a dimensional scaling were true, the position of only one feature of the intensity pattern will characterize the liquid.

\section{The Simple Scaling Assumption}

In order to more clearly distinguish between simple scaling and ordering changes as indicated by the data, we can examine the effects of the scaling assumption on the RDF and on the scattered intensity curves. To do this, we transform the real space variable $r$ to a dimensionless variable $x=r / R(P)$ where $R(P)$ is the position of the first principal peak in the RDF at pressure $P$. The probability function with respect to this new variable is given by $4 \pi x^{2} \rho(x)=R(P) \times$ $4 \pi r^{2} \rho(r)$ when one requires that the number of atoms between $r$ and $(r+d r)$ must be equal to the number between $x$ and $(x+d x)$. This conservation of mass, when applied to the whole system, gives the following relationship between average densities with respect to the two variables: $\rho_{0 x} / \rho_{0 x}=r^{3} / x^{3}$. When this change of variables is applied to the functions in Fig. 5 over the region of the first principal peak, one obtains the scaled radial distribution functions shown in Fig. 7.

The intensity data are commonly presented in terms of the function

$$
i(S)=\frac{I_{1 \mathrm{iq}}^{\mathrm{ooh}} / N-f^{2}}{f^{2}},
$$

which contains the essential information describing the structural variations from uniform density in the liquid and is convenient for theoretical analysis. The function $[i(S)+1]$ is commonly called the liquid structure factor. The $i(S)$ functions for liquid $\mathrm{Na}$ for the four pressures specified above are given in Fig. 8.

Since $S i(S)$ is related to the real space RDF by a Fourier transform as discussed in Sec. V of Paper I, the scaling assumption in real space can be mathematically carried over into the reciprocal $S(=4 \pi \sin \theta / \lambda)$ space. When the function $S i(S)$ is Fourier-transformed to the RDF and scaled and then the scaled RDF is transformed back, one obtains

$$
\operatorname{Si}(S)=Q I(Q) / R(P)
$$

in terms of a reduced reciprocal space vector $Q=$

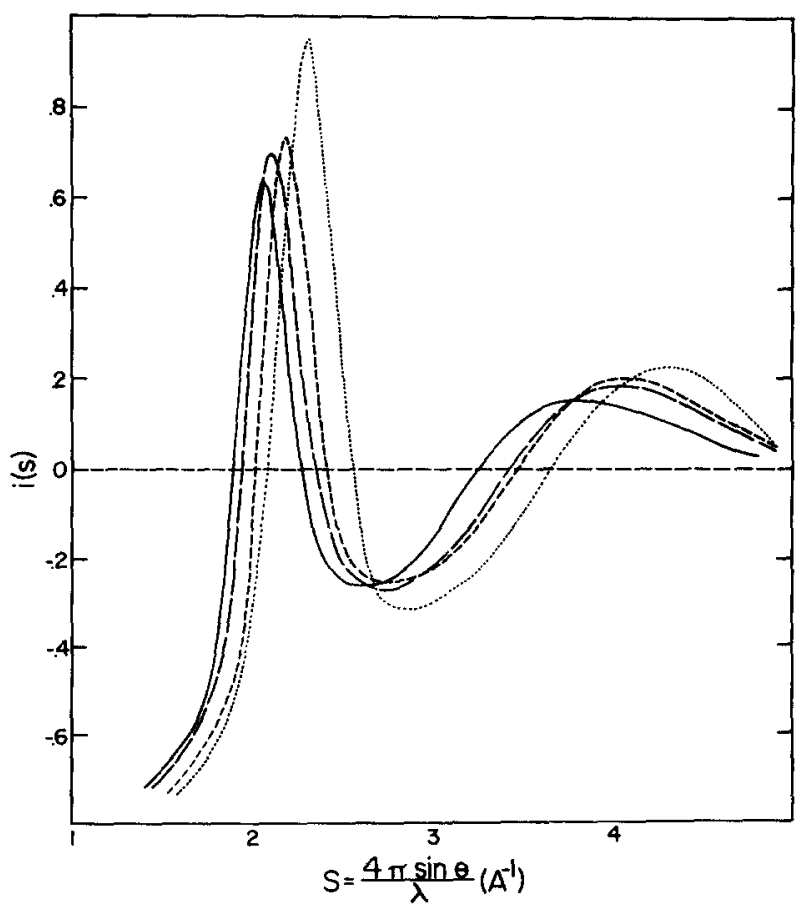

FIG. 8. The function $i(S)$ for $\mathrm{Na}$ at pressures of (a) 0.5 kbar, -; (b) $7.5 \mathrm{kbar}, \ldots-$; (c) $17 \mathrm{kbar}, \ldots$ - ; and (d) $43 \mathrm{kbar}$ -.. 
$S R(P)$. The scaling assumption requires that all radial distribution functions scale to the same function with respect to the reduced variable $x$ and, hence, that all $S i(S)$ functions scale to the same $Q i(Q)$. This allows one to write the function $i(S)$ at pressure $P$, $i_{p}(S)$, in terms of the $i(S)$ function at atmospheric pressure, $i_{0}(S)$, as

$$
i_{p}(S)=i_{0}(a S),
$$

where the scaling constant " $a$ " is given by $a=$ $R(P) / R(0)$. This relationship implies that under the simple scaling assumption, all the $i(S)$ curves shown in Fig. 8 should have the same peak height and simply be stretched or compressed along the $S$ axis. Since the spreading is uniform, the scaling constant $a=R(P) / R(0)$ can be determined from a single point. The position of the first peak, as shown in Fig. 8, is obviously the best defined point and is essentially the same as the position of the first peak in $I^{\mathrm{oomp}}$, which result was used to determine curve (b) in Fig. 6.

\section{Variations from Simple Scaling}

The dominant effect of pressure on liquid sodium along the melting curve, as illustrated in Figs. 6-8, is obviously simple scaling. Departures from simple scaling are indicated by the data but are near the expected limit of the errors introduced by the experiment and subsequent analysis. However, the consistency of the observed variations with pressure leads one to believe that some departure from simple scaling exists. This departure is manifest in at least four ways in the curves in Figs. 6-8: (1) the increasing height and (2) the decreasing width of the first peak in the scaled radial distribution functions in Fig. 7 with pressure, (3) the increasing peak heights in the $i(S)$ functions in Fig. 8 with pressure, and (4) the departure of the observed $\Delta R / R(0)$ values, curve (a) and curve (b) in Fig. 6.

The first three indications of the departure from simple scaling mentioned are directly interrelated. For example, a high first peak in the $i(S)$ function results in a high narrow first peak in the RDF. Absolute values of any of these three quantities are not reliable because of their sensitivity to errors in the analysis procedure and particularly to the magnitude of the $\mathrm{x}$-ray intensity at large angles relative to the height of the first intensity peak. This sensitivity to errors in the large-angle region is enhanced by the normalization procedure which makes $N$ heavily dependent on this region of the intensity and may be the reason for the large difference in the $43 \mathrm{kbar}$ scaled RDF from the others. One would expect higher errors at the higher pressures.

This consistent trend with pressure in the observable effects (1), (2), and (3) mentioned above, coupled with the additional consistency between them, suggests a small but real departure from scaling. This ordering effect indicates that the range in nearest neighbor distances within the liquid is a smaller fraction of the average nearest neighbor distance at high pressures than at one bar. We note for emphasis that scaling would predict a range having the same fraction. This ordering implies a greater curvature in the two-particle potential than that predicted by scaling. In conjunction with this increased ordering, we note that the number of nearest neighbors does not change measurably along the melting curve. Indications are that the packing fraction, however, does change.

If one accepts the fact that the $43 \mathrm{kbar}$ value of $\Delta R / R(0)$ in Fig. 6 lies off the other two curves, this also can be taken as an indication of nonscaling since curve (b) represents an experimental simple scaling approximation. A larger difference between curves (a) and (b) in Fig. 6 would also indicate nonscaling, but the difference shown could well be explained by errors in Pastine's equation of state $^{3}$ or in the assumption of constant entropy change across melting used to obtain (a).

The measurements discussed herein apply only to liquid $\mathrm{Na}$ along the melting curve. High-temperature measurements ${ }^{5,7,9}$ at 1 bar do not indicate a scaling law, and variations are such as to suggest isothermal variations with pressure would depart more drastically from scaling than variations along the melting curve. It would be of interest in conjunction with this data to have measurements off the melting curve, but this data cannot be obtained with the present technique.

We note that the changes observed as a function of pressure along the melting curve are rather sizeable. These changes are several times larger than those reported in the literature due to temperature variation. $5,7,9$ This fact indicates that along the melting curve pressure effects completely dominate temperature effects in terms of interatomic distance.

* This work was supported in part by a grant from the National Science Foundation and the study formed the basis for the doctoral dissertation of K. H. Brown (Brigham Young University, 1969), who was supported by a NASA fellowship during part of the study.

† Present Address: Department of Physics and Earth Sciences, California State Polytechnic College, Pomona, Calif.

1 K. H. Brown and J. D. Barnett, J. Chem. Phys. 57, 2009 (1972), preceding paper.

${ }^{2}$ H. D. Luedemann and G. C. Kennedy, J. Geophys. Res. 73, 2795 (1968).

3 D. J. Pastine, Physical Rev. 166, 703 (1968).

4 R. K. Young, Ph.D. dissertation, Brigham Young University, 1969.

${ }^{5}$ B. R. Orton, B. A. Shaw, and G. I. Williams, Acta Met. 8, 177 (1960).

${ }^{6}$ L. P. Tarasov and B. E. Warren, J. Chem. Phys. 4, 236 (1936)

${ }_{7}^{7}$ F. H. Trimble and N. S. Gingrich, Phys. Rev. 53, 278 (1938).

${ }^{8}$ N. S. Gingrich and L. Heaton, J. Chem. Phys. 34, 873 (1961).

${ }^{2} \mathrm{H}$. Richter and H. Oehme, Z. Naturforsch. 22a, 1470 (1967).

${ }^{10}$ D. L. Decker, Rev. Sci. Instr. 39, 602 (1968).

11 R. N. Jeffery, J. D. Barnett, H. Vanfleet, and H. T. Hall, J. Appl. Phys. 37, 3172 (1966).

12 D. T. Cromer and J. T. Waber, Acta Cryst. 18, 104 (1965).

${ }^{18} \mathrm{D}$. T. Cromer (private communication, 1970).

${ }^{14}$ R. G. Ross, Phil Mag. 22, 573 (1970). 